\title{
$\delta(2,2)$-Invariant for Lagrangian Submanifolds in Quaternionic Space Forms
}

\author{
Gabriel Macsim ${ }^{1,+}+\mathbb{D}$, Adela Mihai ${ }^{2,+}$ (D) and Ion Mihai ${ }^{3, *,+}(\mathbb{C}$ \\ 1 Doctoral School of Mathematics, University of Bucharest, 010014 Bucharest, Romania; \\ gabi_macsim@yahoo.com \\ 2 Department of Mathematics and Computer Science, Technical University of Civil Engineering Bucharest, \\ 020396 Bucharest, Romania; adela.mihai@utcb.ro \\ 3 Department of Mathematics, University of Bucharest, 010014 Bucharest, Romania \\ * Correspondence: imihai@fmi.unibuc.ro \\ + The authors contributed equally to this work.
}

Received: 27 February 2020; Accepted: 23 March 2020; Published: 1 April 2020

check for updates

\begin{abstract}
In the geometry of submanifolds, Chen inequalities represent one of the most important tool to find relationships between intrinsic and extrinsic invariants; the aim is to find sharp such inequalities. In this paper we establish an optimal inequality for the Chen invariant $\delta(2,2)$ on Lagrangian submanifolds in quaternionic space forms, regarded as a problem of constrained maxima.
\end{abstract}

Keywords: $\delta(2,2)$-invariant; Chen inequalities; Lagrangian submanifolds; quaternionic space forms; complex space forms

MSC: 53C40; 53C25

\section{Preliminaries}

In submanifold theory, Lagrangian submanifolds are studied not only for their special geometric properties, but also for their important roles in supersymmetric field theory and string theory. For these submanifolds in quaternionic space forms, we give an answer to one problem in submanifold theory, most precisely to find relationships between the main extrinsic invariants and intrinsic invariants.

The intrinsic characteristics of a Riemannian manifold are given by its curvature invariants. In the second section of this article, we recall the definition of $\delta$-invariants (also known as Chen invariants) (see [1]). This theory was initiated by Chen in [2].

In Section 3 we derive an improved inequality for the Chen invariant $\delta(2,2)$ in the case of a Lagrangian submanifold in a quaternionic space form, regarded as a problem of constrained maxima, and recall the inequality which has been improved.

Let $\widetilde{M}^{m}$ be a complex $m$-dimensional Kaehler manifold endowed with an almost complex structure $J$ and a Hermitian metric $\widetilde{g}$ and $f: M^{n} \rightarrow \widetilde{M}^{m}$ an isometric immersion of an $n$-dimensional manifold $M^{n}$ into $\tilde{M}^{m}$. The submanifold $M^{n}$ is called a totally real submanifold if $J\left(T_{p} M^{n}\right) \subset T_{p}^{\perp} M^{n}, \forall p \in M^{n}$. A totally real submanifold of maximum dimension, i.e., $\operatorname{dim}_{\mathbb{R}} M^{n}=\operatorname{dim}_{\mathbb{C}} \tilde{M}^{n}=n$, is called a Lagrangian submanifold.

If $\widetilde{M}^{m}$ has holomorphic constant sectional curvature $4 c$, then it is called a complex space form and it is denoted by $\widetilde{M}^{m}(4 c)$. Its Riemannian curvature tensor is given by

$$
\widetilde{R}(X, Y) Z=c[g(Y, Z) X-g(X, Z) Y+g(J Y, Z) J X-g(J X, Z) J Y+2 g(X, J Y) J Z]
$$

for any vector fields $X, Y, Z$ tangent to $\tilde{M}^{m}(4 c)$. 
Let $M^{n}$ be a Lagrangian submanifold of $\widetilde{M}^{n}(4 c)$. One denotes by $\nabla$ and $\widetilde{\nabla}$ the Levi-Civita connections of $M^{n}$ and $\widetilde{M}^{n}(4 c)$, respectively. The Gauss and Weingarten formulae are given respectively by

$$
\begin{gathered}
\widetilde{\nabla}_{X} Y=\nabla_{X} Y+h(X, Y), \\
\widetilde{\nabla}_{X} \xi=-A_{\xi} X+D_{X} \xi,
\end{gathered}
$$

where $X$ and $Y$ are tangent vector fields, $\xi$ is a normal vector field and $D$ is the normal connection. The second fundamental form $h$ and shape operator $A_{\tilde{\xi}}$ are related by

$$
g(h(X, Y), \xi)=g\left(A_{\xi} X, Y\right)
$$

The mean curvature vector $H$ of $M^{n}$ is defined by

$$
H=\frac{1}{n} \text { trace } h .
$$

In the case of a Lagrangian submanifold in a complex space form, we have the following relations

$$
\begin{gathered}
D_{X} J Y=J \nabla_{X} Y, \\
A_{J X} Y=-J h(X, Y)=A_{J Y} X,
\end{gathered}
$$

and we point out that $g(h(X, Y), Z)$ is totally symmetric.

One denotes by $K(\pi)$ the sectional curvature of $M^{n}$ associated with a plane section $\pi \subset T_{p} M^{n}$, $p \in M^{n}$ and by $R$ the Riemannian curvature tensor of $M^{n}$. Then the Gauss equation is given by

$$
\begin{aligned}
\widetilde{R}(X, Y, Z, W)= & R(X, Y, Z, W)-g(h(X, Z), h(Y, W))+ \\
& g(h(X, W), h(Y, Z)),
\end{aligned}
$$

for any vectors $X, Y, Z, W$ tangent to $M^{n}$, where $\widetilde{R}(X, Y, Z, W)=g(\widetilde{R}(X, Y) W, Z)$ and $R(X, Y, Z, W)=$ $g(R(X, Y) W, Z)$.

For an orthonormal basis $\left\{e_{1}, e_{2}, \ldots, e_{n}\right\}$ of $T_{p} M^{n}$ at a point $p \in M^{n}$, we put

$$
h_{A B}^{C}=g\left(h\left(e_{A}, e_{B}\right), J e_{C}\right), A, B, C=1, \ldots, n .
$$

Because $g(h(X, Y), Z)$ is totally symmetric, it follows that

$$
h_{B C}^{A}=h_{A C}^{B}=h_{A B}^{C} .
$$

On the other hand, we recall the following result for a Riemannian submanifold $\left(M^{n}, g\right)$ of a Riemannian manifold $\left(\widetilde{M}^{m}, \widetilde{g}\right)$ (of an arbitrary codimension); let consider $f \in C^{\infty}(\widetilde{M})$. We attach the optimum problem:

$$
\min _{x \in M} f(x) .
$$

Then the following result holds (see [3]).

Theorem 1. If $x_{0} \in M^{n}$ is a solution of the problem (8), then

(a) $(\operatorname{grad})\left(x_{0}\right) \in T_{x_{0}}^{\perp} M^{n}$;

(b) the bilinear form $\alpha: T_{x_{0}} M^{n} \times T_{x_{0}} M^{n} \rightarrow \mathbb{R}$,

$$
\alpha(X, Y)=\operatorname{Hess}_{f}(X, Y)+\widetilde{g}\left(h(X, Y),(\operatorname{grad})\left(x_{0}\right)\right)
$$

is semipositive definite, where $h$ is the second fundamental form of the submanifold $M^{n}$ in $\widetilde{M}^{m}$. 


\section{Chen Invariants}

Let $M^{n}$ be an $n$-dimensional Riemannian manifold and $K(\pi)$ the sectional curvature of $M^{n}$ associated with a 2-plane section $\pi \subset T_{p} M^{n}, p \in M^{n}$.

For any orthonormal basis $\left\{e_{1}, \ldots, e_{n}\right\}$ of the tangent space $T_{p} M^{n}$, the scalar curvature $\tau$ at $p$ is defined by

$$
\tau(p)=\sum_{1 \leq i<j \leq n} K\left(e_{i} \wedge e_{j}\right)
$$

One denotes by

$$
(\inf K)(p)=\inf \left\{K(\pi) \mid \pi \subset T_{p} M^{n}, \operatorname{dim} \pi=2\right\} .
$$

The Chen first invariant is given by $\delta_{M}(p)=\tau(p)-(\inf K)(p)$.

If $L$ is a subspace of $T_{p} M^{n}$ of dimension $r \geq 2$ and $\left\{e_{1}, \ldots, e_{r}\right\}$ an orthonormal basis of $L$, the scalar curvature $\tau(L)$ of the $r$-plane section $L$ is given by

$$
\tau(L)=\sum_{1 \leq \alpha<\beta \leq r} K\left(e_{\alpha} \wedge e_{\beta}\right) .
$$

For given integers $n \geq 3$ and $k \geq 1$, one denotes by $S(n, k)$ the finite set of all $k$-tuples $\left(n_{1}, \ldots, n_{k}\right)$ of integers satisfying $2 \leq n_{1}, \ldots, n_{k}<n, n_{1}+\ldots+n_{k} \leq n$. Let $S(n)=\bigcup_{k \geq 1} S(n, k)$.

For each $\left(n_{1}, \ldots, n_{k}\right) \in S(n)$ and each point $p \in M^{n}$, B.-Y. Chen introduced a Riemannian invariant defined by

$$
\delta\left(n_{1}, \ldots, n_{k}\right)(p)=\tau(p)-\inf \left\{\tau\left(L_{1}\right)+\ldots+\tau\left(L_{k}\right)\right\}
$$

where $L_{1}, \ldots, L_{k}$ run over all $k$ mutually orthogonal subspaces of $T_{p} M^{n}$ such that $\operatorname{dim} L_{j}=n_{j}, j=1, \ldots, k$.

We recall the most important Chen inequalities for submanifolds in real space forms.

Theorem 2 ([2]). Let $M^{n}$ be an $n$-dimensional $(n \geq 3)$ submanifold of a real space form $\tilde{M}^{m}(c)$ of constant sectional curvature $c$. Then

$$
\delta_{M} \leq \frac{n-2}{2}\left\{\frac{n^{2}}{n-1}\|H\|^{2}+(n+1) c\right\}
$$

The equality case was characterized in terms of the shape operator.

The same inequality holds for totally real submanifolds in complex space forms. A corresponding inequality for slant submanifolds in complex space forms was obtained in [4].

However, for Lagrangian submanifolds in complex space forms the above inequality, known as Chen first inequality, was improved by Bolton et al. [5]. Moreover, one of the present authors improved the Chen first inequality for Kaehlerian slant submanifolds in complex space forms (see [6]).

For each $\left(n_{1}, \ldots, n_{k}\right) \in S(n)$, one denotes by:

$$
\begin{gathered}
d\left(n_{1}, \ldots, n_{k}\right)=\frac{n^{2}\left(n+k-1-\sum_{j=1}^{k} n_{j}\right)}{2\left(n+k-\sum_{j=1}^{k} n_{j}\right)}, \\
b\left(n_{1}, \ldots, n_{k}\right)=\frac{1}{2}\left[n(n-1)-\sum_{j=1}^{k} n_{j}\left(n_{j}-1\right)\right] .
\end{gathered}
$$

The following sharp inequality involving the Chen invariants and the squared mean curvature obtained in [7] plays a very important role in this topic. 
Theorem 3. For each $\left(n_{1}, \ldots, n_{k}\right) \in S(n)$ and each $n$-dimensional submanifold $M^{n}$ in a Riemannian space form $\widetilde{M}^{m}(4 c)$ of constant sectional curvature $4 c$, the inequality

$$
\delta\left(n_{1}, \ldots, n_{k}\right) \leq d\left(n_{1}, \ldots, n_{k}\right)\|H\|^{2}+b\left(n_{1}, \ldots, n_{k}\right) c
$$

is fulfilled.

Chen also pointed-out that a similar inequality holds for totally real (in particular Lagrangian) submanifolds in a complex space form.

\section{Lagrangian Submanifolds in Quaternionic Space Forms}

Chen et al. established the following inequalities for Chen invariants of Lagrangian submanifolds in complex space forms, which improve the inequality (10).

Theorem 4 ([8]). Let $M^{n}$ be a Lagrangian submanifold of a complex space form $\widetilde{M}^{n}(4 c)$. For a given $k$-tuple $\left(n_{1}, n_{2}, \ldots, n_{k}\right) \in S(n)$, we put $N=n_{1}+n_{2}+\ldots+n_{k}$. If $N<n$, then the inequality

$$
\begin{aligned}
\delta\left(n_{1}, n_{2}, \ldots, n_{k}\right) \leq & \frac{n^{2}\left\{n-N+3 k-1-6 \sum_{i=1}^{k}\left(2+n_{i}\right)^{-1}\right\}}{2\left\{n-N+3 k+2-6 \sum_{i=1}^{k}\left(2+n_{i}\right)^{-1}\right\}}\|H\|^{2}+ \\
& \frac{1}{2}\left\{n(n-1)-\sum_{i=1}^{k} n_{i}\left(n_{i}-1\right)\right\} c
\end{aligned}
$$

is satisfied.

In particular, one has (see also [9]).

Theorem 5. Let $M^{n}$ be a Lagrangian submanifold of a complex space form $\widetilde{M}^{n}(4 c), n \geq 4$. Then the following inequality holds.

$$
\delta(2,2) \leq \frac{n^{2}}{2} \cdot \frac{n-2}{n+1}\|H\|^{2}+\frac{1}{2}[n(n-1)-4] c .
$$

The equality sign holds at a point $p \in M^{n}$ if and only if there is an orthonormal basis $\left\{e_{1}, e_{2}, \ldots, e_{n}\right\}$ at $p$ such that with respect to this basis the second fundamental form $h$ satisfies the following conditions

$$
\begin{gathered}
h_{i A}^{C}=0, A, C \in\{1, \ldots, n\} \backslash\{i\}, A<C, i=\overline{1,3}, \\
h_{B C}^{A}=0, A=\overline{1, n}, 4 \leq B<C \leq n, A \notin\{B, C\} .
\end{gathered}
$$

Next, we recall some basic notions about quaternionic space forms.

Let $\widetilde{M}^{4 m}$ be a differentiable manifold and we assume that there is a rank 3 subbundle $\sigma$ of $\operatorname{End}\left(T \widetilde{M}^{4 m}\right)$ such that a local basis $\left\{J_{1}, J_{2}, J_{3}\right\}$ exists on sections of $\sigma$ satisfying for all $\alpha \in\{1,2,3\}$

$$
J_{\alpha}^{2}=-\mathrm{Id}, \quad J_{\alpha} J_{\alpha+1}=-J_{\alpha+1} J_{\alpha}=J_{\alpha+2},
$$

where Id denotes the identity field of type $(1,1)$ on $\widetilde{M}^{4 m}$ and the indices are taken from $\{1,2,3\}$ modulo 3. The bundle $\sigma$ is called an almost quaternionic structure on $\widetilde{M}^{4 m}$ and $\left\{J_{1}, J_{2}, J_{3}\right\}$ is called a canonical basis of $\sigma$. $\left(\widetilde{M}^{4 m}, \sigma\right)$ is said to be an almost quaternionic manifold. It is easy to see that any almost quaternionic manifold is of dimension $4 m, m \geq 1$.

A Riemannian metric $\widetilde{g}$ on $\widetilde{M}^{4 m}$ is said to be adapted to the almost quaternionic structure $\sigma$ if it satisfies

$$
\widetilde{g}\left(J_{\alpha} X, J_{\alpha} Y\right)=\widetilde{g}(X, Y), \quad \forall \alpha \in\{1,2,3\},
$$


for all vector fields $X, Y$ on $\widetilde{M}^{4 m}$ and any canonical basis $\left\{J_{1}, J_{2}, J_{3}\right\}$ on $\sigma .\left(\widetilde{M}^{4 m}, \sigma, \widetilde{g}\right)$ is said to be an almost quaternionic Hermitian manifold.

$\left(\widetilde{M}^{4 m}, \sigma, \widetilde{g}\right)$ is said to be a quaternionic Kaehler manifold if the bundle $\sigma$ is parallel with respect to the Levi-Civita connection $\widetilde{\nabla}$ of $\widetilde{g}$, i.e., locally defined 1-forms $\omega_{1}, \omega_{2}, \omega_{3}$ exist such that we have

$$
\widetilde{\nabla}_{X} J_{\alpha}=\omega_{\alpha+2}(X) J_{\alpha+1}-\omega_{\alpha+1}(X) J_{\alpha+2}
$$

for all $\alpha \in\{1,2,3\}$ and for any vector field $X$ on $\widetilde{M}^{4 m}$, where the indices are taken from $\{1,2,3\}$ modulo 3 .

Let $\left(\widetilde{M}^{4 m}, \sigma, \widetilde{g}\right)$ be a quaternionic Kaehler manifold and let $X$ be a non-null vector on $\widetilde{M}^{4 m}$. The 4-plane spanned by $\left\{X, J_{1} X, J_{2} X, J_{3} X\right\}$ is called a quaternionic 4-plane and is denoted by $Q(X)$. Any 2-plane in $Q(X)$ is called a quaternionic plane. The sectional curvature of a quaternionic plane is called a quaternionic sectional curvature. A quaternionic Kaehler manifold is a quaternionic space form if its quaternionic sectional curvature are equal to a constant, say $4 c$, i.e., its curvature tensor is given by

$$
\begin{aligned}
\widetilde{R}(X, Y) Z= & c\left\{\widetilde{g}(Z, Y) X-\widetilde{g}(X, Z) Y+\sum_{\alpha=1}^{3}\left[\widetilde{g}\left(Z, J_{\alpha} Y\right) J_{\alpha} X-\right.\right. \\
& \left.\left.\widetilde{g}\left(Z, J_{\alpha} X\right) J_{\alpha} Y+2 \widetilde{g}\left(X, J_{\alpha} y\right) J_{\alpha} Z\right]\right\},
\end{aligned}
$$

for all vector fields $X, Y, Z$ on $\widetilde{M}^{4 m}$ and any local basis $\left\{J_{1}, J_{2}, J_{3}\right\}$ on $\sigma$.

A submanifold $M^{n}$ of a quaternionic space form $\widetilde{M}^{4 n}(4 c)$ is said to be Lagrangian if $J_{\alpha}\left(T_{p} M\right) \subset$ $T_{p}^{\perp} M$, for any $p \in M$ and $\alpha=1,2,3$.

On a Lagrangian submanifold $M^{n}$ we can choose an orthonormal frame field in $\widetilde{M}^{4 n}(4 c)$

$$
\begin{gathered}
\left\{e_{1}, e_{2}, \ldots, e_{n} ; \quad e_{\phi_{1}(1)}=J_{1}\left(e_{1}\right), \ldots, e_{\phi_{1}(n)}=J_{1}\left(e_{n}\right) ;\right. \\
\left.e_{\phi_{2}(1)}=J_{2}\left(e_{1}\right), \ldots, e_{\phi_{2}(n)}=J_{2}\left(e_{n}\right) ; \quad e_{\phi_{3}(1)}=J_{3}\left(e_{1}\right), \ldots, e_{\phi_{3}(n)}=J_{3}\left(e_{n}\right)\right\},
\end{gathered}
$$

such that, restricted to $M, e_{1}, e_{2}, \ldots, e_{n}$ are tangent to $M$.

We set

$$
h_{i j}^{\xi}=g\left(h\left(e_{i}, e_{j}\right), e_{\xi}\right), \quad \xi \in\left\{\phi_{1}(1), \ldots, \phi_{1}(n), \phi_{2}(1), \ldots, \phi_{2}(n), \phi_{3}(1), \ldots, \phi_{3}(n)\right\}
$$

and then, for any $\alpha=1,2,3$, we have (see (2.9) in [10])

$$
h_{i j}^{\phi_{\alpha}(k)}=h_{k i}^{\phi_{\alpha}(j)}=h_{j k}^{\phi_{\alpha}(i)} .
$$

We denote by $H^{r}=g\left(H, e_{\phi_{1}\left(e_{r}\right)}\right)$, for $r=1, \ldots, n$.

By using the method of constrained maxima, we prove the following improved Chen inequality for the invariant $\delta(2,2)$ of Lagrangian submanifolds in quaternionic space forms, the main result of this paper.

Theorem 6. Let $M^{n}$ be a Lagrangian submanifold of a quaternionic space form $\widetilde{M}^{4 n}(4 c), n \geq 4$. Then the inequality

$$
\delta(2,2) \leq \frac{n^{2}}{2} \cdot \frac{n-2}{n+1}\|H\|^{2}+\frac{1}{2}[n(n-1)-4] c
$$

is fulfilled.

The equality sign holds at a point $p \in M^{n}$ if and only if there is an orthonormal basis $\left\{e_{1}, e_{2}, \ldots, e_{n}\right\}$ at $p$ such that with respect to this basis the second fundamental form $h$ satisfies the following conditions:

$$
h_{i A}^{\phi_{\alpha}(C)}=0, A, C \in\{1, \ldots, n\} \backslash\{i\}, A<C, i=\overline{1,3}, \alpha=\overline{1,3},
$$




$$
h_{B C}^{\phi_{\alpha}(A)}=0, A=\overline{1, n}, 4 \leq B<C \leq n, A \notin\{B, C\}, \alpha=\overline{1,3} .
$$

Proof. Let $M^{n}$ be a Lagrangian submanifold of the quaternionic space form $\widetilde{M}^{4 n}(4 c), p \in M^{n}$ and $L_{1}$ and $L_{2}$ two mutual orthogonal plane sections at $p$. We denote $\left\{e_{1}, e_{2}\right\} \subset L_{1},\left\{e_{3}, e_{4}\right\} \subset L_{2}$ orthonormal bases, complete to an orthonormal basis $\left\{e_{1}, \ldots, e_{n}\right\} \subset T_{p} M^{n}$ and extend it to $T_{p} \widetilde{M}^{4 n}(4 c)$ as above.

Gauss equation implies

$$
\begin{gathered}
\tau=\sum_{\alpha=1}^{3} \sum_{A=1}^{n} \sum_{B<C}\left[h_{B B}^{\phi_{\alpha}(A)} h_{C C}^{\phi_{\alpha}(A)}-\left(h_{B C}^{\phi_{\alpha}(A)}\right)^{2}\right]+\frac{n(n-1)}{2} c, \\
\tau\left(L_{1}\right)=\sum_{\alpha=1}^{3} \sum_{A=1}^{n}\left[h_{11}^{\phi_{\alpha}(A)} h_{22}^{\phi_{\alpha}(A)}-\left(h_{12}^{\phi_{\alpha}(A)}\right)^{2}\right]+c, \\
\tau\left(L_{2}\right)=\sum_{\alpha=1}^{3} \sum_{A=1}^{n}\left[h_{33}^{\phi_{\alpha}(A)} h_{44}^{\phi_{\alpha}(A)}-\left(h_{34}^{\phi_{\alpha}(A)}\right)^{2}\right]+c .
\end{gathered}
$$

Then

$$
\begin{gathered}
\tau-\tau\left(L_{1}\right)-\tau\left(L_{2}\right)-[n(n-1)-4] \frac{C}{2}= \\
\sum_{\alpha=1}^{3} \sum_{A=1}^{n} \sum_{B<C}\left[h_{B B}^{\phi_{\alpha}(A)} h_{C C}^{\phi_{\alpha}(A)}-\left(h_{B C}^{\phi_{\alpha}(A)}\right)^{2}\right]- \\
\sum_{\alpha=1}^{3} \sum_{A=1}^{n}\left[h_{11}^{\phi_{\alpha}(A)} h_{22}^{\phi_{\alpha}(A)}+h_{33}^{\phi_{\alpha}(A)} h_{44}^{\phi_{\alpha}(A)}-\left(h_{12}^{\phi_{\alpha}(A)}\right)^{2}-\left(h_{34}^{\phi_{\alpha}(A)}\right)^{2}\right]= \\
\sum_{\alpha=1}^{3} \sum_{A=1}^{n}\left[\sum_{1 \leq B<C \leq n} h_{B B}^{\phi_{\alpha}(A)} h_{C C}^{\phi_{\alpha}(A)}-h_{11}^{\phi_{\alpha}(A)} h_{22}^{\phi_{\alpha}(A)}-h_{33}^{\phi_{\alpha}(A)} h_{44}^{\phi_{\alpha}(A)}\right]- \\
\sum_{\alpha=1}^{3} \sum_{A=1}^{n}\left[\sum_{1 \leq B<C \leq n}\left(h_{B C}^{\phi_{\alpha}(A)}\right)^{2}-\left(h_{12}^{\phi_{\alpha}(A)}\right)^{2}-\left(h_{34}^{\phi_{\alpha}(A)}\right)^{2}\right] .
\end{gathered}
$$

Thus, we get

$$
\tau-\tau\left(L_{1}\right)-\tau\left(L_{2}\right)-[n(n-1)-4] \frac{c}{2} \leq
$$

$\sum_{\alpha=1}^{3} \sum_{A=1}^{n}\left[\left(h_{11}^{\phi_{\alpha}(A)}+h_{22}^{\phi_{\alpha}(A)}\right) \sum_{B=3}^{n} h_{B B}^{\phi_{\alpha}(A)}+\left(h_{33}^{\phi_{\alpha}(A)}+h_{44}^{\phi_{\alpha}(A)}\right) \sum_{B=5}^{n} h_{B B}^{\phi_{\alpha}(A)}+\sum_{5 \leq B<C \leq n} h_{B B}^{\phi_{\alpha}(A)} h_{C C}^{\phi_{\alpha}(A)}\right]-$

$$
\begin{gathered}
\sum_{\alpha=1}^{3} \sum_{B=3}^{n}\left[\left(h_{1 B}^{\phi_{\alpha}(1)}\right)^{2}+\left(h_{1 B}^{\phi_{\alpha}(B)}\right)^{2}+\left(h_{2 B}^{\phi_{\alpha}(2)}\right)^{2}+\left(h_{2 B}^{\phi_{\alpha}(B)}\right)^{2}\right]-\sum_{\alpha=1}^{3} \sum_{B=5}^{n}\left[\left(h_{3 B}^{\phi_{\alpha}(3)}\right)^{2}+\left(h_{3 B}^{\phi_{\alpha}(B)}\right)^{2}\right]- \\
\sum_{\alpha=1}^{3} \sum_{4 \leq B<C \leq n}\left[\left(h_{B C}^{\phi_{\alpha}(B)}\right)^{2}+\left(h_{B C}^{\phi_{\alpha}(C)}\right)^{2}\right] .
\end{gathered}
$$

It follows that

$$
\tau-\tau\left(L_{1}\right)-\tau\left(L_{2}\right)-[n(n-1)-4] \frac{c}{2} \leq
$$

$\sum_{\alpha=1}^{3} \sum_{A=1}^{n}\left[\left(h_{11}^{\phi_{\alpha}(A)}+h_{22}^{\phi_{\alpha}(A)}\right) \sum_{B=3}^{n} h_{B B}^{\phi_{\alpha}(A)}+\left(h_{33}^{\phi_{\alpha}(A)}+h_{44}^{\phi_{\alpha}(A)}\right) \sum_{B=5}^{n} h_{B B}^{\phi_{\alpha}(A)}+\sum_{5 \leq B<C \leq n} h_{B B}^{\phi_{\alpha}(A)} h_{C C}^{\phi_{\alpha}(A)}\right]-$ 


$$
\begin{gathered}
\sum_{\alpha=1}^{3} \sum_{B=3}^{n}\left[\left(h_{11}^{\phi_{\alpha}(B)}\right)^{2}+\left(h_{B B}^{\phi_{\alpha}(1)}\right)^{2}+\left(h_{22}^{\phi_{\alpha}(B)}\right)^{2}+\left(h_{B B}^{\phi_{\alpha}(2)}\right)^{2}\right]-\sum_{\alpha=1}^{3} \sum_{B=5}^{n}\left[\left(h_{33}^{\phi_{\alpha}(B)}\right)^{2}+\left(h_{B B}^{\phi_{\alpha}(3)}\right)^{2}\right]- \\
\sum_{\alpha=1}^{3} \sum_{4 \leq B<C \leq n}\left[\left(h_{B B}^{\phi_{\alpha}(C)}\right)^{2}+\left(h_{C C}^{\phi_{\alpha}(B)}\right)^{2}\right] .
\end{gathered}
$$

For each $\alpha \in\{1,2,3\}$, let us consider certain quadratic forms.

For example, for $\alpha=1$, we will define the quadratic forms

$$
f_{1}, f_{2}, \ldots, f_{n}: \mathbb{R}^{n} \rightarrow \mathbb{R}
$$

by

$$
\begin{gathered}
f_{r}\left(h_{11}^{\phi_{1}(r)}, h_{22}^{\phi_{1}(r)}, \ldots, h_{n n}^{\phi_{1}(r)}\right)=\left(h_{11}^{\phi_{1}(r)}+h_{22}^{\phi_{1}(r)}\right) \sum_{B=3}^{n} h_{B B}^{\phi_{1}(r)}+\left(h_{33}^{\phi_{1}(r)}+h_{44}^{\phi_{1}(r)}\right) \sum_{B=5}^{n} h_{B B}^{\phi_{1}(r)}+ \\
\sum_{5 \leq B<C \leq n} h_{B B}^{\phi_{1}(r)} h_{C C}^{\phi_{1}(r)}-\sum_{B=3}^{n}\left(h_{B B}^{\phi_{1}(r)}\right)^{2},
\end{gathered}
$$

for $r=1,2$,

$$
\begin{gathered}
f_{s}\left(h_{11}^{\phi_{1}(s)}, h_{22}^{\phi_{1}(s)}, \ldots, h_{n n}^{\phi_{1}(s)}\right)=\left(h_{11}^{\phi_{1}(s)}+h_{22}^{\phi_{1}(s)}\right) \sum_{B=3}^{n} h_{B B}^{\phi_{1}(s)}+\left(h_{33}^{\phi_{1}(s)}+h_{44}^{\phi_{1}(s)}\right) \sum_{B=5}^{n} h_{B B}^{\phi_{1}(s)}+ \\
\sum_{5 \leq B<C \leq n} h_{B B}^{\phi_{1}(s)} h_{C C}^{\phi_{1}(s)}-\left(h_{11}^{\phi_{1}(s)}\right)^{2}-\left(h_{22}^{\phi_{1}(s)}\right)^{2}-\sum_{B=5}^{n}\left(h_{B B}^{\phi_{1}(s)}\right)^{2},
\end{gathered}
$$

for $s=3,4$,

$$
\begin{gathered}
f_{t}\left(h_{11}^{\phi_{1}(t)}, h_{22}^{\phi_{1}(t)}, \ldots, h_{n n}^{\phi_{1}(t)}\right)=\left(h_{11}^{\phi_{1}(t)}+h_{22}^{\phi_{1}(t)}\right) \sum_{B=3}^{n} h_{B B}^{\phi_{1}(t)}+\left(h_{33}^{\phi_{1}(t)}+h_{44}^{\phi_{1}(t)}\right) \sum_{B=5}^{n} h_{B B}^{\phi_{1}(t)}+ \\
\sum_{5 \leq B<C \leq n} h_{B B}^{\phi_{1}(t)} h_{C C}^{\phi_{1}(t)}-\sum_{B=1 ; B \neq t}^{n}\left(h_{B B}^{\phi_{1}(t)}\right)^{2},
\end{gathered}
$$

for $5 \leq t \leq n$.

We shall find an upper bound for $f_{1}$, subject to

$$
P: h_{11}^{\phi_{1}(1)}+h_{22}^{\phi_{1}(1)}+\ldots+h_{n n}^{\phi_{1}(1)}=k^{1}
$$

where $k^{1}$ is a real number.

Let $q \in P$ an arbitrary point. The bilinear form $\gamma: T_{q} P \times T_{q} P \rightarrow \mathbb{R}$ has the expression

$$
\gamma(X, Y)=\operatorname{Hess}\left(f_{r}\right)(X, Y)+\left\langle h^{\prime}(X, Y), \operatorname{grad} f_{r}(q)\right\rangle
$$

where $h^{\prime}$ is the second fundamental form of $P$ in $\mathbb{R}^{n}$ and $\langle$,$\rangle is the standard inner-product on \mathbb{R}^{n}$.

The partial derivatives of the function $f_{1}$ are

$$
\frac{\partial f_{1}}{\partial h_{11}^{\phi_{1}(1)}}=\sum_{B=3}^{n} h_{B B}^{\phi_{1}(1)},
$$




$$
\begin{gathered}
\frac{\partial f_{1}}{\partial h_{22}^{\phi_{1}(1)}}=\sum_{B=3}^{n} h_{B B}^{\phi_{1}(1)}, \\
\frac{\partial f_{1}}{\partial h_{r r}^{\phi_{1}(1)}}=h_{11}^{\phi_{1}(1)}+h_{22}^{\phi_{1}(1)}+\sum_{B=5}^{n} h_{B B}^{\phi_{1}(1)}-2 h_{r r}^{\phi_{1}(1)}, r=3,4, \\
\frac{\partial f_{1}}{\partial h_{t t}^{\phi_{1}(1)}}=\sum_{B=1}^{n} h_{B B}^{\phi_{1}(1)}-3 h_{t t}^{\phi_{1}(1)}, 5 \leq t \leq n .
\end{gathered}
$$

In the standard frame of $\mathbb{R}^{n}$, the Hessian of $f_{1}$ has the matrix

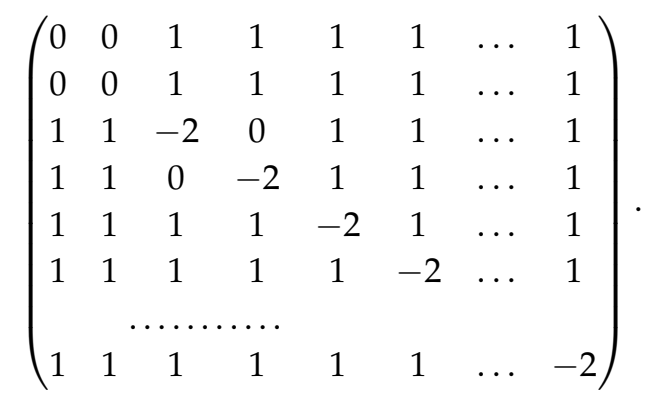

As $P$ is totally geodesic in $\mathbb{R}^{n}$, we obtain

$$
\begin{aligned}
\gamma(X, X)= & 2\left(X_{1}+X_{2}\right) \sum_{i=3}^{n} X_{i}+2\left(X_{3}+X_{4}\right) \sum_{i=5}^{n} X_{i}+2 \sum_{5 \leq i<j \leq n} X_{i} X_{j}-2 \sum_{i=3}^{n}\left(X_{i}\right)^{2}= \\
& \left(\sum_{i=1}^{n} X_{i}\right)^{2}-\left(X_{1}\right)^{2}-\left(X_{2}\right)^{2}-3 \sum_{i=3}^{n}\left(X_{i}\right)^{2}-2 X_{1} X_{2}-2 X_{3} X_{4}= \\
& -\left(X_{1}+X_{2}\right)^{2}-\left(X_{3}+X_{4}\right)^{2}-2\left(X_{3}\right)^{2}-2\left(X_{4}\right)^{2}-3 \sum_{i=5}^{n}\left(X_{i}\right)^{2}<0
\end{aligned}
$$

then the Hessian of $f_{1}$ is negative semidefinite.

Searching for the critical point $h_{11}^{\phi_{1}(1)}, h_{22}^{\phi_{1}(1)}, \ldots, h_{n n}^{\phi_{1}(1)}$ of $f_{1}$, we denote by

$$
h_{33}^{\phi_{1}(1)}=h_{44}^{\phi_{1}(1)}=a^{1} .
$$

Then,

$$
\begin{gathered}
4 h_{33}^{\phi_{1}(1)}=3 h_{r r}^{\phi_{1}(1)}, r=\overline{5, n} \Longrightarrow h_{r r}^{\phi_{1}(1)}=\frac{4 a^{1}}{3}, 5 \leq r \leq n, \\
h_{11}^{\phi_{1}(1)}+h_{22}^{\phi_{1}(1)}=3 h_{t t}^{\phi_{1}(1)}, t=\overline{5, n} \Longrightarrow h_{11}^{\phi_{1}(1)}+h_{22}^{\phi_{1}(1)}=4 a^{1} .
\end{gathered}
$$

From (20) it follows that

$$
4 a^{1}+2 a^{1}+\frac{4 a^{1}}{3}(n-4)=k^{1} \Longrightarrow a^{1}=\frac{3 k^{1}}{2(2 n+1)} .
$$

This implies

$$
\begin{gathered}
h_{11}^{\phi_{1}(1)}+h_{22}^{\phi_{1}(1)}=\frac{6 k^{1}}{2 n+1^{\prime}}, \\
h_{33}^{\phi_{1}(1)}=h_{44}^{\phi_{1}(1)}=\frac{3 k^{1}}{2(2 n+1)}, \\
h_{r r}^{\phi_{1}(1)}=\frac{2 k^{1}}{2 n+1}, 5 \leq r \leq n .
\end{gathered}
$$


Thus

$$
\begin{aligned}
& f_{1} \leq \frac{6 k^{1}}{2 n+1}\left[\frac{6 k^{1}}{4 n+2}+(n-4) \frac{2 k^{1}}{2 n+1}\right]+\frac{3 k^{1}}{2 n+1}(n-4) \frac{2 k^{1}}{2 n+1}+ \\
& C_{n-4}^{2}\left(\frac{2 k^{1}}{2 n+1}\right)^{2}-2\left(\frac{3 k^{1}}{4 n+2}\right)^{2}-(n-4)\left(\frac{2 k^{1}}{2 n+1}\right)^{2}= \\
& \frac{6 k^{1}}{2 n+1} \cdot\left[\frac{3 k^{1}}{2 n+1}+(n-4) \frac{2 k^{1}}{2 n+1}\right]+\frac{3 k^{1}}{2(2 n+1)}(n-4) \frac{2 k^{1}}{2 n+1}+ \\
& \frac{3 k^{1}}{2(2 n+1)}(n-4) \frac{2 k^{1}}{2 n+1}+C_{n-4}^{2}\left(\frac{2 k^{1}}{2 n+1}\right)^{2}-2 \frac{\left(3 k^{1}\right)^{2}}{4(2 n+1)^{2}}-(n-4) \frac{\left(2 k^{1}\right)^{2}}{(2 n+1)^{2}}= \\
& \frac{6 k^{1}}{2 n+1} \cdot \frac{3 k^{1}+2(n-4) k^{1}}{2 n+1}+\frac{3(n-4)\left(k^{1}\right)^{2}}{(2 n+1)^{2}}+\frac{3(n-4)\left(k^{1}\right)^{2}}{(2 n+1)^{2}}+ \\
& \frac{(n-4)(n-5)}{2} \cdot \frac{4\left(k^{1}\right)^{2}}{(2 n+1)^{2}}-\frac{9\left(k^{1}\right)^{2}}{2(2 n+1)^{2}}-\frac{4(n-4)\left(k^{1}\right)^{2}}{(2 n+1)^{2}}= \\
& \frac{6 k^{1}}{2 n+1} \cdot \frac{(2 n-5) k^{1}}{(2 n+1)}+\frac{6(n-4)\left(k^{1}\right)^{2}}{(2 n+1)^{2}}+\frac{2(n-4)(n-5)\left(k^{1}\right)^{2}}{(2 n+1)^{2}}- \\
& \frac{9\left(k^{1}\right)^{2}}{2(2 n+1)^{2}}-\frac{4(n-4)\left(k^{1}\right)^{2}}{(2 n+1)^{2}}= \\
& \frac{\left(k^{1}\right)^{2}}{(2 n+1)^{2}} \cdot\left[6(2 n-5)+6(n-4)+2(n-4)(n-5)-\frac{9}{2}-4(n-4)\right]= \\
& \frac{\left(k^{1}\right)^{2}}{(2 n+1)^{2}} \cdot\left[12 n-30+6 n-24+(2 n-8)(n-5)-\frac{9}{2}-4 n+16\right]= \\
& \frac{\left(k^{1}\right)^{2}}{2(2 n+1)^{2}} \cdot\left(24 n-60+12 n-48+4 n^{2}-20 n-16 n+80-9-8 n+32\right)= \\
& \frac{\left(k^{1}\right)^{2}}{2(2 n+1)^{2}} \cdot\left(4 n^{2}-8 n-5\right)=\frac{\left(k^{1}\right)^{2}}{2(2 n+1)^{2}} \cdot(2 n-5)(2 n+1),
\end{aligned}
$$

which implies

$$
f_{1} \leq \frac{(2 n-5)\left(k^{1}\right)^{2}}{2(2 n+1)}
$$

i.e.,

$$
f_{1} \leq \frac{n^{2}}{2} \cdot \frac{2 n-5}{2 n+1}\left(H^{1}\right)^{2} .
$$

In a similar manner, we obtain for $f_{2}$

$$
f_{2} \leq \frac{n^{2}}{2} \cdot \frac{2 n-5}{2 n+1}\left(H^{2}\right)^{2} .
$$

Let's consider now $f_{3}$, as:

$$
\begin{gathered}
f_{3}\left(h_{11}^{\phi_{1}(3)}, h_{22}^{\phi_{1}(3)}, \ldots, h_{n n}^{\phi_{1}(3)}\right)=\left(h_{11}^{\phi_{1}(3)}+h_{22}^{\phi_{1}(3)}\right) \sum_{B=3}^{n} h_{B B}^{\phi_{1}(3)}+\left(h_{33}^{\phi_{1}(3)}+h_{44}^{\phi_{1}(3)}\right) \sum_{B=5}^{n} h_{B B}^{\phi_{1}(3)}+ \\
\sum_{5 \leq B<C \leq n} h_{B B}^{\phi_{1}(3)} h_{C C}^{\phi_{1}(3)}-\left(h_{11}^{\phi_{1}(3)}\right)^{2}-\left(h_{22}^{\phi_{1}(3)}\right)^{2}-\sum_{B=5}^{n}\left(h_{B B}^{\phi_{1}(3)}\right)^{2} .
\end{gathered}
$$


The partial derivatives of the function $f_{3}$ are

$$
\begin{gathered}
\frac{\partial f_{3}}{\partial h_{r r}^{\phi_{1}(3)}}=\sum_{B=3}^{n} h_{B B}^{\phi_{1}(3)}-2 h_{r r}^{\phi_{1}(3)}, r=1,2, \\
\frac{\partial f_{3}}{\partial h_{s s}^{\phi_{1}(3)}}=h_{11}^{\phi_{1}(3)}+h_{22}^{\phi_{1}(3)}+\sum_{B=5}^{n} h_{B B}^{\phi_{1}(3)}, s=3,4, \\
\frac{\partial f_{3}}{\partial h_{t t}^{\phi_{1}(3)}}=\sum_{B=1}^{n} h_{B B}^{\phi_{1}(3)}-3 h_{t t}^{\phi_{1}(3)}, 5 \leq t \leq n .
\end{gathered}
$$

In the standard frame of $\mathbb{R}^{n}$, the Hessian of $f_{3}$ has the matrix

$$
\left(\begin{array}{cccccccc}
-2 & 0 & 1 & 1 & 1 & 1 & \ldots & 1 \\
0 & -2 & 1 & 1 & 1 & 1 & \ldots & 1 \\
1 & 1 & 0 & 0 & 1 & 1 & \ldots & 1 \\
1 & 1 & 0 & 0 & 1 & 1 & \ldots & 1 \\
1 & 1 & 1 & 1 & -2 & 1 & \ldots & 1 \\
1 & 1 & 1 & 1 & 1 & -2 & \ldots & 1 \\
7 & \ldots & \ldots & \ldots & & & & \\
1 & 1 & 1 & 1 & 1 & 1 & \ldots & -2
\end{array}\right)
$$

As $P$ is totally geodesic in $\mathbb{R}^{n}$, we have

$$
\begin{gathered}
\gamma(X, X)=-2\left[\left(X_{1}\right)^{2}+\left(X_{2}\right)^{2}+\sum_{i=5}^{n}\left(X_{i}\right)^{2}\right]+ \\
2\left(X_{1}+X_{2}\right) \sum_{i=3}^{n} X_{i}+2\left(X_{3}+X_{4}\right) \sum_{i=5}^{n} X_{i}+2 \sum_{5 \leq i<j \leq n} X_{i} X_{j}= \\
\left(\sum_{i=1}^{n} X_{i}\right)^{2}-2 X_{1} X_{2}-2 X_{3} X_{4}-\sum_{i=1}^{n}\left(X_{i}\right)^{2}-2\left(X_{1}\right)^{2}-2\left(X_{2}\right)^{2}-2 \sum_{i=5}^{n}\left(X_{i}\right)^{2}= \\
\left(\sum_{i=1}^{n} X_{i}\right)^{2}-\left(X_{1}+X_{2}\right)^{2}-\left(X_{3}+X_{4}\right)^{2}-2\left(X_{1}\right)^{2}-2\left(X_{2}\right)^{2}-3 \sum_{i=5}^{n}\left(X_{i}\right)^{2}<0,
\end{gathered}
$$

and hence the Hessian of $f_{3}$ is negative semidefinite.

If we denote by $q=\left(h_{11}^{\phi_{1}(3)}, h_{22}^{\phi_{1}(3)}, \ldots, h_{n n}^{\phi_{1}(3)}\right)$ a solution of the extremum problem in question, then we have

$$
\begin{gathered}
h_{11}^{\phi_{1}(3)}=h_{22}^{\phi_{1}(3)}, \\
h_{55}^{\phi_{1}(3)}=h_{66}^{\phi_{1}(3)}=\ldots=h_{n n}^{\phi_{1}(3)}, \\
4 h_{11}^{\phi_{1}(3)}=3 h_{55}^{\phi_{1}(3)}, \\
h_{33}^{\phi_{1}(3)}+h_{44}^{\phi_{1}(3)}=3 h_{55}^{\phi_{1}(3)} .
\end{gathered}
$$

Thus

$$
h_{33}^{\phi_{1}(3)}+h_{44}^{\phi_{1}(3)}=4 h_{11}^{\phi_{1}(3)} .
$$

Considering

$$
h_{55}^{\phi_{1}(3)}=h_{66}^{\phi_{1}(3)}=\ldots=h_{n n}^{\phi_{1}(3)}=a^{3},
$$


we obtain

$$
\begin{aligned}
& h_{11}^{\phi_{1}(3)}=h_{22}^{\phi_{1}(3)}=\frac{3 a^{3}}{4} \\
& h_{33}^{\phi_{1}(3)}+h_{44}^{\phi_{1}(3)}=3 a^{3} .
\end{aligned}
$$

Since $h_{11}^{\phi_{1}(3)}+h_{22}^{\phi_{1}(3)}+\ldots+h_{n n}^{\phi_{1}(3)}=k^{3}$, then $a^{3}=\frac{2 k^{3}}{2 n+1}$, which implies

$$
\begin{gathered}
h_{11}^{\phi_{1}(3)}=h_{22}^{\phi_{1}(3)}=\frac{3 k^{3}}{2(2 n+1)}, \\
h_{33}^{\phi_{1}(3)}+h_{44}^{\phi_{1}(3)}=\frac{6 k^{3}}{2 n+1}, \\
h_{55}^{\phi_{1}(3)}=h_{66}^{\phi_{1}(3)}=\ldots=h_{n n}^{\phi_{1}(3)}=\frac{2 k^{3}}{2 n+1} .
\end{gathered}
$$

It follows that

$$
\begin{gathered}
f_{3} \leq 2 \cdot \frac{3 k^{3}}{2(2 n+1)} \cdot\left[\frac{6 k^{3}}{2 n+1}+(n-4) \frac{2 k^{3}}{2 n+1}\right]+\frac{6 k^{3}}{2 n+1}(n-4) \frac{2 k^{3}}{2 n+1}+ \\
C_{n-4}^{2} \cdot \frac{\left(2 k^{3}\right)^{2}}{(2 n+1)^{2}}-2 \frac{\left(3 k^{3}\right)^{2}}{4(2 n+1)^{2}}-(n-4)\left(\frac{2 k^{3}}{2 n+1}\right)^{2}= \\
\frac{3 k^{3}}{(2 n+1)} \cdot \frac{6 k^{3}+2(n-4) k^{3}}{(2 n+1)}+\frac{12(n-4)\left(k^{3}\right)^{2}}{(2 n+1)^{2}}+ \\
\frac{(n-4)(n-5)}{2} \cdot \frac{4\left(k^{3}\right)^{2}}{(2 n+1)^{2}}-\frac{9\left(k^{3}\right)^{2}}{2(2 n+1)^{2}}-\frac{4(n-4)\left(k^{3}\right)^{2}}{(2 n+1)^{2}}= \\
\frac{\left(k^{3}\right)^{2}}{(2 n+1)^{2}} \cdot\left[3(2 n-2)+12(n-4)+2(n-4)(n-5)-\frac{9}{2}-4(n-4)\right]= \\
\frac{\left(k^{3}\right)^{2}}{(2 n+1)^{2}} \cdot\left(6 n-6+12 n-48+2 n^{2}-10 n-8 n+40-\frac{9}{2}-4 n+16\right)= \\
\frac{\left(k^{3}\right)^{2}}{2(2 n+1)^{2}} \cdot\left(12 n-12+24 n-96+4 n^{2}-20 n-16 n+80-9-8 n+32\right),
\end{gathered}
$$

or, equivalently,

$$
f_{3} \leq \frac{\left(k^{3}\right)^{2}\left(4 n^{2}-8 n-5\right)}{2(2 n+1)^{2}}=\frac{\left(k^{3}\right)^{2}(2 n-5)(2 n+1)}{2(2 n+1)^{2}}
$$

Therefore,

$$
f_{3} \leq \frac{n^{2}}{2} \cdot \frac{2 n-5}{2 n+1}\left(H^{3}\right)^{2} .
$$

In a similar manner, we prove for $f_{4}$ :

$$
f_{4} \leq \frac{n^{2}}{2} \cdot \frac{2 n-5}{2 n+1}\left(H^{4}\right)^{2} .
$$


Using the same procedure for

$$
\begin{gathered}
f_{5}\left(h_{11}^{\phi_{1}(5)}, h_{22}^{\phi_{1}(5)}, \ldots, h_{n n}^{\phi_{1}(5)}\right)=\left(h_{11}^{\phi_{1}(5)}+h_{22}^{\phi_{1}(5)}\right) \sum_{B=3}^{n} h_{B B}^{\phi_{1}(5)}+\left(h_{33}^{\phi_{1}(5)}+h_{44}^{\phi_{1}(5)}\right) \sum_{B=5}^{n} h_{B B}^{\phi_{1}(5)}+ \\
\sum_{5 \leq B<C \leq n} h_{B B}^{\phi_{1}(5)} h_{C C}^{\phi_{1}(5)}-\sum_{B=1 ; B \neq 5}^{n}\left(h_{B B}^{\phi_{1}(5)}\right)^{2}
\end{gathered}
$$

we find the partial derivatives of $f_{5}$

$$
\begin{gathered}
\frac{\partial f_{5}}{\partial h_{r r}^{\phi_{1}(5)}}=\sum_{B=3}^{n} h_{B B}^{\phi_{1}(5)}-2 h_{r r}^{\phi_{1}(5)}, r=1,2, \\
\frac{\partial f_{5}}{\partial h_{s s}^{\phi_{1}(5)}}=h_{11}^{\phi_{1}(5)}+h_{22}^{\phi_{1}(5)}+\sum_{B=5}^{n} h_{B B}^{\phi_{1}(5)}-2 h_{s s}^{\phi_{1}(5)}, s=3,4, \\
\frac{\partial f_{5}}{\partial h_{55}^{\phi_{1}(5)}}=\sum_{B=1}^{n} h_{B B}^{\phi_{1}(5)}-h_{55}^{\phi_{1}(5)}, \\
\frac{\partial f_{5}}{\partial h_{t t}^{\phi_{1}(5)}}=\sum_{B=1}^{n} h_{B B}^{\phi_{1}(5)}-3 h_{t t}^{\phi_{1}(5)}, 6 \leq t \leq n .
\end{gathered}
$$

In the standard frame of $\mathbb{R}^{n}$, the Hessian of $f_{5}$ has the matrix

$$
\left(\begin{array}{cccccccc}
-2 & 0 & 1 & 1 & 1 & 1 & \ldots & 1 \\
0 & -2 & 1 & 1 & 1 & 1 & \ldots & 1 \\
1 & 1 & -2 & 0 & 1 & 1 & \ldots & 1 \\
1 & 1 & 0 & -2 & 1 & 1 & \ldots & 1 \\
1 & 1 & 1 & 1 & 0 & 1 & \ldots & 1 \\
1 & 1 & 1 & 1 & 1 & -2 & \ldots & 1 \\
7 & \ldots \ldots \ldots \ldots & & & & \\
1 & 1 & 1 & 1 & 1 & 1 & \ldots & -2
\end{array}\right)
$$

As $P$ is totally geodesic in $\mathbb{R}^{n}$, we have

$$
\begin{gathered}
\gamma(X, X)=-2 \sum_{i=1 ; i \neq 5}^{n}\left(X_{i}\right)^{2}+ \\
2\left(X_{1}+X_{2}\right) \sum_{i=3}^{n} X_{i}+2\left(X_{3}+X_{4}\right) \sum_{i=5}^{n} X_{i}+2 \sum_{5 \leq i<j \leq n} X_{i} X_{j}= \\
\left(\sum_{i=1}^{n} X_{i}\right)^{2}-2 X_{1} X_{2}-2 X_{3} X_{4}-\sum_{i=1}^{n}\left(X_{i}\right)^{2}-2 \sum_{i=1 ; i \neq 5}^{n}\left(X_{i}\right)^{2}= \\
\left(\sum_{i=1}^{n} X_{i}\right)^{2}-\left(X_{1}+X_{2}\right)^{2}-\left(X_{3}+X_{4}\right)^{2}-\left(X_{5}\right)^{2}-2 \sum_{i=1}^{4}\left(X_{i}\right)^{2}-3 \sum_{i=6}^{n}\left(X_{i}\right)^{2}<0,
\end{gathered}
$$

and hence the Hessian of $f_{5}$ is negative semidefinite.

Using similar arguments to those in the previous problem we obtain that the solution of the associated extremum problem is

$$
h_{11}^{\phi_{1}(5)}=h_{22}^{\phi_{1}(5)}=h_{33}^{\phi_{1}(5)}=h_{44}^{\phi_{1}(5)}=3 a^{5},
$$




$$
\begin{gathered}
h_{55}^{\phi_{1}(5)}=12 a^{5}, \\
h_{66}^{\phi_{1}(5)}=\ldots=h_{n n}^{\phi_{1}(5)}=4 a^{5},
\end{gathered}
$$

where $a^{5}$ is a real number.

Since $h_{11}^{\phi_{1}(5)}+h_{22}^{\phi_{1}(5)}+\ldots+h_{n n}^{\phi_{1}(5)}=k^{5}$, then $a^{5}=\frac{k^{5}}{4(n+1)}$ and

$$
\begin{gathered}
h_{1}^{\phi_{1}(5)}=h_{22}^{\phi_{1}(5)}=h_{33}^{\phi_{1}(5)}=h_{44}^{\phi_{1}(5)}=\frac{3 k^{5}}{4(n+1)}, \\
h_{55}^{\phi_{1}(5)}=\frac{3 k^{5}}{n+1}, \\
h_{66}^{\phi_{1}(5)}=\ldots=h_{n n}^{\phi_{1}(5)}=\frac{k^{5}}{n+1} .
\end{gathered}
$$

We have

$$
\begin{aligned}
& f_{5} \leq \frac{6 k^{5}}{4(n+1)} \cdot\left[\frac{6 k^{5}}{4(n+1)}+\frac{3 k^{5}}{n+1}+(n-5) \frac{k^{5}}{n+1}\right]+ \\
& 2 \cdot \frac{3 k^{5}}{4(n+1)}\left[\frac{3 k^{5}}{n+1}+(n-5) \frac{k^{5}}{n+1}\right]+ \\
& \frac{3 k^{5}}{n+1}(n-5) \frac{k^{5}}{n+1}+C_{n-5}^{2} \frac{\left(k^{5}\right)^{2}}{(n+1)^{2}}-4 \cdot \frac{\left(3 k^{5}\right)^{2}}{16(n+1)^{2}}-(n-5) \frac{\left(k^{5}\right)^{2}}{(n+1)^{2}}= \\
& \frac{3 k^{5}}{2(n+1)} \cdot\left[\frac{3 k^{5}}{2(n+1)}+\frac{3 k^{5}}{n+1}+\frac{(n-5) k^{5}}{n+1}\right]+\frac{3 k^{5}}{2(n+1)} \cdot\left[\frac{3 k^{5}+(n-5) k^{5}}{n+1}\right]+ \\
& \frac{3(n-5)\left(k^{5}\right)^{2}}{(n+1)^{2}}+\frac{(n-5)(n-6)}{2} \cdot \frac{\left(k^{5}\right)^{2}}{(n+1)^{2}}-\frac{9\left(k^{5}\right)^{2}}{4(n+1)^{2}}-\frac{(n-5)\left(k^{5}\right)^{2}}{(n+1)^{2}}= \\
& \frac{\left(k^{5}\right)^{2}}{4(n+1)^{2}} \cdot 3(9+2 n-10)+\frac{\left(k^{5}\right)^{2}}{2(n+1)^{2}} \cdot 3(3+n-5)+\frac{3(n-5)\left(k^{5}\right)^{2}}{(n+1)^{2}}+ \\
& \frac{(n-5)(n-6)\left(k^{5}\right)^{2}}{2(n+1)^{2}}-\frac{9\left(k^{5}\right)^{2}}{4(n+1)^{2}}-\frac{(n-5)\left(k^{5}\right)^{2}}{(n+1)^{2}}= \\
& \frac{\left(k^{5}\right)^{2}}{(n+1)^{2}}\left[\frac{3(2 n-1)}{4}+\frac{3(n-2)}{2}+3(n-5)+\frac{(n-5)(n-6)}{2}-\frac{9}{4}-(n-5)\right]= \\
& \frac{\left(k^{5}\right)^{2}}{4(n+1)^{2}}[3(2 n-1)+6(n-2)+12(n-5)+(2 n-10)(n-6)-9-4(n-5)]= \\
& \frac{\left(k^{5}\right)^{2}}{4(n+1)^{2}}\left(6 n-3+6 n-12+12 n-60+2 n^{2}-12 n-10 n+60-9-4 n+20\right)= \\
& \frac{\left(k^{5}\right)^{2}}{4(n+1)^{2}}\left(2 n^{2}-2 n-4\right)=\frac{\left(k^{5}\right)^{2}}{2(n+1)^{2}}\left(n^{2}-n-2\right)=\frac{\left(k^{5}\right)^{2}}{2(n+1)^{2}}(n+1)(n-2) .
\end{aligned}
$$

From this we get

$$
f_{5} \leq \frac{\left(k^{5}\right)^{2}}{2} \cdot \frac{n-2}{n+1}
$$

or, equivalently,

$$
f_{5} \leq \frac{n^{2}}{2} \cdot\left(\frac{n-2}{n+1}\right)\left(H^{5}\right)^{2}
$$


In the same manner we prove for $f_{r}$, with $5 \leq r \leq n$,

$$
f_{r} \leq \frac{n^{2}}{2} \cdot\left(\frac{n-2}{n+1}\right)\left(H^{r}\right)^{2}
$$

Applying this procedure for each $\alpha \in\{1,2,3\}$ and taking into account that

$$
\frac{2 n-5}{2 n+1}<\frac{n-2}{n+1}
$$

we find

$$
\delta(2,2) \leq \frac{n^{2}}{2} \cdot \frac{n-2}{n+1}\|H\|^{2}+[n(n-1)-4] \frac{c}{2},
$$

which is the inequality to prove.

Remark 1. In [11], the first author obtained certain Chen inequalities for Lagrangian submanifolds $M^{n}$ in quaternionic space forms $\widetilde{M}^{4 n}(4 c)$. In particular, for the Chen invariant $\delta(2,2)$ one derives the inequality

$$
\delta(2,2) \leq \frac{n^{2}(n-1)}{2(n+2)}\|H\|^{2}+\frac{1}{2}[n(n-1)-4] c .
$$

We want to point-out that the inequality from Theorem 6 improves the inequality (21) because $\frac{n-2}{n+1}<$ $\frac{n-1}{n+2}$, for $n>4$.

Author Contributions: Conceptualization, G.M.; methodology, A.M.; software, G.M.; validation, G.M., A.M. and I.M.; formal analysis, I.M.; investigation, G.M., A.M. and I.M.; writing-review and editing, G.M. and A.M.; supervision, I.M. All authors have read and agreed to the published version of the manuscript.

Funding: This research received no external funding.

Conflicts of Interest: The authors declare no conflict of interest.

\section{References}

1. Chen, B.-Y. $\delta$-Invariants, Inequalities of Submanifolds and Their Applications. In Topics in Differential Geometry; Mihai, A., Mihai, I., Miron, R., Eds.; Editura Academiei Române: Bucharest, Romania, 2008; pp. 29-155.

2. Chen, B.-Y. Some pinching and classification theorems for minimal submanifolds. Arch. Math. 1993, 60, 568-578. [CrossRef]

3. Oprea, T. Optimizations on Riemannian submanifolds. Analele Univ. Buc. 2005, 54, 127-136.

4. Oiagă, A.; Mihai, I.B.Y. Chen inequalities for slant submanifolds in complex space forms. Demonstratio Math. 1999, 32, 835-846. [CrossRef]

5. Bolton, J.; Dillen, F.; Fastenakels, J.; Vrancken, L. A best possible inequality for curvature-like tensor fields. Math. Inequal. Appl. 2009, 12, 663-681. [CrossRef]

6. Mihai, A. Geometric inequalities for purely real submanifolds in complex space forms. Results Math. 2009, 55, 457-468. [CrossRef]

7. Chen, B.-Y. Some new obstructions to minimal and Lagrangian isometric immersions. Jpn. J. Math. 2000, 26, 105-127. [CrossRef]

8. Chen, B.-Y.; Dillen, F.; Van der Veken, J.; Vrancken, L. Curvature inequalities for Lagrangian submanifolds: The final solution. Diff. Geom. Appl. 2013, 31, 808-819. [CrossRef]

9. Chen, B.-Y.; Prieto, A.; Wang, X. Lagrangian submanifolds in complex space forms satisfying an improved equality involving $\delta(2,2)$. Publ. Math. Debrecen 2013, 82, 193-217. [CrossRef] 
10. Chen, B.-Y.; Houh, C.S. Totally real submanifolds of a quaternion projective space. Ann. Mat. Pura Appl. 1979, 120, 185-199. [CrossRef]

11. Macsim, G. Improved Chen's inequalities for Lagrangian submanifolds in quaternionic space forms. Romanian J. Math. Comp. Sci. 2016, 6, 61-84.

(C) 2020 by the authors. Licensee MDPI, Basel, Switzerland. This article is an open access article distributed under the terms and conditions of the Creative Commons Attribution (CC BY) license (http:/ / creativecommons.org/licenses/by/4.0/). 